\title{
Fixed-Point Theorems for Multivalued Mappings in Modular Metric Spaces
}

\section{Parin Chaipunya, Chirasak Mongkolkeha, Wutiphol Sintunavarat, and Poom Kumam}

Department of Mathematics, Faculty of Science, King Mongkut's University of Technology Thonburi (KMUTT), Bangkok 10140, Thailand

Correspondence should be addressed to Poom Kumam, poom.kum@kmutt.ac.th

Received 7 December 2011; Accepted 20 January 2012

Academic Editor: Josef Diblík

Copyright (C) 2012 Parin Chaipunya et al. This is an open access article distributed under the Creative Commons Attribution License, which permits unrestricted use, distribution, and reproduction in any medium, provided the original work is properly cited.

We give some initial properties of a subset of modular metric spaces and introduce some fixedpoint theorems for multivalued mappings under the setting of contraction type. An appropriate example is as well provided. The stability of fixed points in our main theorems is also studied.

\section{Introduction and Preliminaries}

The field of metric fixed-point theory has been widely investigated since 1922, when Banach [1] had proved his contraction principle. We are going to recall this well-known theorem before we continue over on.

A self-mapping $f$ on a metric space $(X, d)$ is called a contraction if there exists $0 \leq k<1$ such that

$$
d(f x, f y) \leq k d(x, y)
$$

for all $x, y \in X$. The contraction principle simply stated that, if $(X, d)$ is complete, such a mapping has a unique fixed point.

One of the most influenced generalizations of Banach's theorem is traced to Nadler [2]. In 1969, via Hausdorff's concept of a distance between two arbitrary sets, Nadler proved the contraction principle for multivalued mappings in complete metric spaces. Also, some authors extended Nadler's principle and established fixed-point theorems for multivalued mappings in metric spaces and other spaces (see [3-9]). One of the most interesting studies 
are the extensions of such principle in modular spaces and modular function spaces (see [10-12] and references therein).

Lately, in 2010, Chistyakov [13] introduced the notion of a modular metric space which is a new generalization of a metric space. We will give a short revisit to modular and modular metric spaces as follows.

Definition 1.1. Let $X$ be a linear space over $\mathbb{R}$ with $\theta \in X$ as its zero element. A functional $\rho$ : $X \rightarrow[0,+\infty]$ is said to be a modular on $X$ if for any $x, y \in X$, the following conditions hold:

(i) $\rho(x)=0$ if and only if $x=\theta$,

(ii) $\rho(x)=\rho(-x)$,

(iii) $\rho(\alpha x+\beta y) \leq \rho(x)+\rho(y)$ whenever $\alpha, \beta \geq 0$ and $\alpha+\beta=1$.

The linear subspace $X_{\rho}:=\left\{x \in X: \lim _{\lambda \rightarrow 0} \rho(\lambda x)=0\right\}$ is called a modular space.

Definition 1.2 (see [13]). Let $X$ be a nonempty set. A function $\omega:(0,+\infty) \times X \times X \rightarrow[0,+\infty]$ is said to be a metric modular on $X$ if satisfying, for all $x, y, z \in X$, the following conditions hold:

(i) $\omega_{\lambda}(x, y)=0$ for all $\lambda>0$ if and only if $x=y$,

(ii) $\omega_{\lambda}(x, y)=\omega_{\lambda}(y, x)$ for all $\lambda>0$,

(iii) $\omega_{\lambda+\mu}(x, y) \leq \omega_{\lambda}(x, z)+\omega_{\mu}(z, y)$ for all $\lambda, \mu>0$.

Suppose $x_{\iota} \in X$, the set $X_{\omega}\left(x_{\iota}\right)=\left\{x \in X: \lim _{\lambda \rightarrow+\infty} \omega_{\lambda}\left(x, x_{\iota}\right)=0\right\}$ is called a modular metric space generated by $x_{\iota}$ and induced by $\omega$. If its generator $x_{\iota}$ does not play any role in the situation, we will write $X_{\omega}$ instead of $X_{\omega}\left(x_{t}\right)$.

Observe that a metric modular $\omega$ on $X$ is nonincreasing with respect to $\lambda>0$. We can simply show this assertion by using the condition (iii) itself. For any $x, y \in X$ and $0<\mu<\lambda$, we have

$$
\omega_{\lambda}(x, y) \leq \omega_{\lambda-\mu}(x, x)+\omega_{\mu}(x, y)=\omega_{\mu}(x, y)
$$

For each $x, y \in X$ and $\lambda>0$, we set $\omega_{\lambda^{+}}(x, y):=\lim _{\epsilon \downarrow 0} \omega_{\lambda+\epsilon}(x, y)$ and $\omega_{\lambda^{-}}(x, y):=\lim _{\epsilon \downarrow 0} \omega_{\lambda-\epsilon}$ $(x, y)$. Consequently, from (1.2), we have $\omega_{\lambda^{+}}(x, y) \leq \omega_{\lambda}(x, y) \leq \omega_{\lambda^{-}}(x, y)$.

If, for any $x, y \in X$, a metric modular $\omega$ on $X$ possesses a finite value and $\omega_{\lambda}(x, y)=$ $\omega_{\mu}(x, y)$ for all $\lambda, \mu>0$, then $d(x, y):=\omega_{\lambda}(x, y)$ is a metric on $X$.

Recently, Mongkolkeha et al. [14] have introduced some notions and established some fixed-point results in modular metric spaces. We now state some notions and results in [14] in the following.

Definition 1.3 (see [14]). Let $X_{\omega}$ be a modular metric space.

(i) The sequence $\left\{x_{n}\right\}_{n \in \mathbb{N}}$ in $X_{\omega}$ is said to be convergent if there exists $x \in X_{\omega}$ such that $\omega_{\lambda}\left(x_{n}, x\right) \rightarrow 0$, as $n \rightarrow \infty$ for all $\lambda>0$.

(ii) The sequence $\left\{x_{n}\right\}_{n \in \mathbb{N}}$ in $X_{\omega}$ is said to be a Cauchy sequence if $\omega_{\lambda}\left(x_{m}, x_{n}\right) \rightarrow 0$, as $m, n \rightarrow \infty$ for all $\lambda>0$.

(iii) $X_{\omega}$ is said to be complete if every Cauchy sequence in $X_{\omega}$ converges. 
(iv) A subset $C$ of $X_{\omega}$ is said to be closed if the limit of a convergent sequence of $C$ always belongs to $C$.

(v) A subset $C$ of $X_{\omega}$ is said to be bounded if, for all $\lambda>0, \phi_{\lambda}(C)=\sup \left\{\omega_{\lambda}(x, y): x, y \in\right.$ C $\}<+\infty$.

Along this paper, we will use the following alternative notions of convergence and Cauchyness, which are equivalent to the notions given above.

Let $X_{\omega}$ be a modular metric space and $\left\{x_{n}\right\}_{n \in \mathbb{N}}$ be a sequence in $X_{\omega}$.

(i) A point $x \in X_{\omega}$ is called a limit of $\left\{x_{n}\right\}_{n \in \mathbb{N}}$ if for each $\lambda, \epsilon>0$, there exists $n_{0} \in \mathbb{N}$ such that $\omega_{\lambda}\left(x_{n}, x\right)<\epsilon$ for every $n \in \mathbb{N}$ with $n \geq n_{0}$. A sequence that has a limit is said to be convergent (or converges to $x$ ) and will be written as $\lim _{n \rightarrow+\infty} x_{n}=x$.

(ii) A sequence $\left\{x_{n}\right\}_{n \in \mathbb{N}}$ in $X_{\omega}$ is said to be a Cauchy sequence if, for each $\lambda, \epsilon>0$, there exists $n_{0} \in \mathbb{N}$ such that $\omega_{\lambda}\left(x_{n}, x_{m}\right)<\epsilon$ for every $m, n \in \mathbb{N}$ with $m, n \geq n_{0}$.

Moreover, we observe that the limit of any sequence in $X_{\omega}$ is unique.

Definition 1.4 (see [14]). Let $X_{\omega}$ be a modular metric space. A self-mapping $f$ on $X_{\omega}$ is said to be a contraction if there exists $0 \leq k<1$ such that

$$
\omega_{\lambda}(f x, f y) \leq k \omega_{\lambda}(x, y)
$$

for all $x, y \in X_{\omega}$ and $\lambda>0$.

Theorem 1.5 (see [14]). Let $X_{\omega}$ be a complete modular metric space and $f$ a contraction on $X_{\omega}$. Then, the sequence $\left\{f^{n} x\right\}_{n \in \mathbb{N}}$ converges to the unique fixed point of $f$ in $X_{\omega}$ for any initial $x \in X_{\omega}$.

The purpose of this paper is to study some properties of a subset of modular metric spaces, establish and extend some fixed-point theorems of Mongkolkeha et al. [14] to multivalued mappings in modular metric spaces.

\section{Some Properties of a Subset of Modular Metric Spaces}

In this section, we study some properties of a subset of modular metric spaces, some of which will take advantages in the proof of our main theorems. Throughout this paper, let $\mathcal{C} B\left(X_{\omega}\right)$ denotes the set of all nonempty closed bounded subsets of $X_{\omega}$ and $\mathcal{C}(X)$ denotes the set of all nonempty closed subsets of $X$.

Let $A$ be a non-empty subset of a modular metric space $X_{\omega}$. For $x \in X_{\omega}$, we denotes $\omega_{\lambda}(x, A):=\inf _{y \in A} \omega_{\lambda}(x, y)$.

For $A, B \in \mathcal{C} B\left(X_{\omega}\right)$, define $\delta_{\lambda}(A, B):=\sup _{x \in A} \omega_{\lambda}(x, B)$ and the Hausdorff metric modular $\Omega_{\lambda}(A, B):=\max \left\{\delta_{\lambda}(A, B), \delta_{\lambda}(B, A)\right\}$. Notice that $\delta_{\lambda}$ is not symmetric.

Proposition 2.1. Let $X_{\omega}$ be a modular metric space and $A, B, C \in \mathcal{C} B\left(X_{\omega}\right)$. Then, the following properties hold.

(i) $\delta_{\lambda}(A, B)=0$ for all $\lambda>0 \Leftrightarrow A \subseteq B$.

(ii) $B \subseteq C \Rightarrow \delta_{\lambda}(A, C) \leq \delta_{\lambda}(A, B)$ for all $\lambda>0$.

(iii) $\delta_{\lambda}(A \cup B, C)=\max \left\{\delta_{\lambda}(A, C), \delta_{\lambda}(B, C)\right\}$ for all $\lambda>0$.

(iv) $\delta_{\lambda+\mu}(A, B) \leq \delta_{\lambda}(A, C)+\delta_{\mu}(C, B)$ for all $\lambda, \mu>0$. 
Proof. (i) By the definition of $\delta_{\lambda}$, we have, for all $\lambda>0$, that

$$
\begin{aligned}
\delta_{\lambda}(A, B)=0 & \Longleftrightarrow \sup _{x \in A} \omega_{\lambda}(x, B)=0 \\
& \Longleftrightarrow \omega_{\lambda}(x, B)=0, \quad \forall x \in A .
\end{aligned}
$$

Since $B$ is closed in $X_{\omega}$, we get $\omega_{\lambda}(x, B)=0$ for all $\lambda>0 \Leftrightarrow x \in B$. That is, $\delta_{\lambda}(A, B)=0$ for all $\lambda>0 \Leftrightarrow A \subseteq B$. $\delta_{\lambda}(A, B)$.

(ii) It is obvious that $\omega_{\lambda}(x, C) \leq \omega_{\lambda}(x, B)$ for all $x \in X_{\omega}$ and $\lambda>0$. Hence, $\delta_{\lambda}(A, C) \leq$

(iii) Observe that, if $B \subseteq C$, then

$$
\delta_{\lambda}(A \cup B, C)=\sup _{x \in A \cup B} \omega_{\lambda}(x, C)=\max \left\{\sup _{x \in A} \omega_{\lambda}(x, C), \sup _{x \in B} \omega_{\lambda}(x, C)\right\} .
$$

(iv) Let $a \in A, b \in B$, and $c \in C$. Then,

$$
\omega_{\lambda+\mu}(a, b) \leq \omega_{\lambda}(a, c)+\omega_{\mu}(c, b)
$$

which implies that

$$
\begin{aligned}
\omega_{\lambda+\mu}(a, B) & \leq \omega_{\lambda}(a, c)+\omega_{\mu}(c, B) \\
& \leq \omega_{\lambda}(a, c)+\delta_{\mu}(C, B) .
\end{aligned}
$$

Since $c \in C$ is arbitrary, we have

$$
\omega_{\lambda+\mu}(a, B) \leq \omega_{\lambda}(a, C)+\delta_{\mu}(C, B) .
$$

Similarly, since $a \in A$ is arbitrary, we can deduce that

$$
\delta_{\lambda+\mu}(A, B) \leq \delta_{\lambda}(A, C)+\delta_{\mu}(C, B) .
$$

Proposition 2.2. Let $X_{\omega}$ be a modular metric space. Then,

$$
\Omega_{\lambda}(A \cup B, C \cup D) \leq \max \left\{\Omega_{\lambda}(A, C), \Omega_{\lambda}(B, D)\right\}
$$

for all $A, B, C, D \in \mathcal{C} B\left(X_{\omega}\right)$. 
Proof. Suppose $\lambda>0$ is arbitrary. For $a \in A$ and $b \in B$, we have $\omega_{\lambda}(a, C \cup D) \leq \omega_{\lambda}(a, C)$ and $\omega_{\lambda}(b, C \cup D) \leq \omega_{\lambda}(b, D)$. Hence, we get

$$
\begin{aligned}
\delta_{\lambda}(A \cup B, C \cup D) & =\max \left\{\delta_{\lambda}(A, C \cup D), \delta_{\lambda}(B, C \cup D)\right\} \\
& \leq \max \left\{\delta_{\lambda}(A, C), \delta_{\lambda}(B, D)\right\} \\
& \leq \max \left\{\Omega_{\lambda}(A, C), \Omega_{\lambda}(B, D)\right\} .
\end{aligned}
$$

Similarly, we have

$$
\delta_{\lambda}(C \cup D, A \cup B) \leq \max \left\{\Omega_{\lambda}(A, C), \Omega_{\lambda}(B, D)\right\}
$$

Hence, we have

$$
\begin{aligned}
\Omega_{\lambda}(A \cup B, C \cup D) & =\max \left\{\delta_{\lambda}(A \cup B, C \cup D), \delta_{\lambda}(C \cup D, A \cup B)\right\} \\
& \leq \max \left\{\Omega_{\lambda}(A, C), \Omega_{\lambda}(B, D)\right\} .
\end{aligned}
$$

Proposition 2.3. Let $X_{\omega}$ be a modular metric space generated by $x_{\iota}$. Then, $\mathrm{CB}\left(X_{\omega}\right)$ is a modular metric space generated by $\left\{x_{l}\right\}$ and is induced by $\Omega$.

Proof. For $\left\{x_{\iota}\right\}, A \in \mathcal{C} B\left(X_{\omega}\right)$, we have

$$
\begin{aligned}
\Omega_{\lambda}\left(A,\left\{x_{\iota}\right\}\right) & =\max \left\{\sup _{x \in A} \omega_{\lambda}\left(x,\left\{x_{\iota}\right\}\right), \sup _{x \in\left\{x_{\iota}\right\}} \omega_{\lambda}(x, A)\right\} \\
& =\max \left\{\sup _{x \in A} \omega_{\lambda}\left(x, x_{\iota}\right), \inf _{x \in A} \omega_{\lambda}\left(x_{\iota}, x\right)\right\} \\
& =\sup _{x \in A} \omega_{\lambda}\left(x, x_{\iota}\right) .
\end{aligned}
$$

Since $x \in A \subseteq X_{\omega}$ and $\lim _{\lambda \rightarrow+\infty} w_{\lambda}\left(x, x_{l}\right)=0$, we have $\lim _{\lambda \rightarrow+\infty} \Omega_{\lambda}\left(A,\left\{x_{l}\right\}\right)=0$.

By the definition of $\Omega$ and Proposition 2.1, it is clear that $\Omega_{\lambda}(A, B)=\Omega_{\lambda}(B, A) \geq 0$ for all $\lambda>0$ and $\Omega_{\lambda}(A, B)=0$ for all $\lambda>0$ if and only if $A=B$.

Again, by Proposition 2.1, we have

$$
\begin{aligned}
\Omega_{\lambda+\mu}(A, B) & =\max \left\{\delta_{\lambda+\mu}(A, B), \delta_{\lambda+\mu}(B, A)\right\} \\
& \leq \max \left\{\delta_{\lambda}(A, C)+\delta_{\mu}(C, B), \delta_{\mu}(B, C)+\delta_{\lambda}(C, A)\right\} \\
& \leq \max \left\{\delta_{\lambda}(A, C), \delta_{\lambda}(C, A)\right\}+\max \left\{\delta_{\mu}(B, C), \delta_{\mu}(C, B)\right\} \\
& =\Omega_{\lambda}(A, C)+\Omega_{\mu}(C, B)
\end{aligned}
$$

for all $\lambda, \mu>0$. Therefore, $\mathcal{C B}\left(X_{\omega}\right)$ is a modular metric space generated by $\left\{x_{\ell}\right\}$ and is induced by $\Omega$. 
Remark 2.4. Note that the metric modular $\Omega$ depends on $\omega$, so the completeness of $X_{\omega}$ implies the completeness of $\mathcal{C B}\left(X_{\omega}\right)$.

Now, we are arriving at the most important lemma used in our proof of main theorems.

Lemma 2.5. Let $A, B \in \mathcal{C} B\left(X_{\omega}\right)$ and $a \in A$. Then, for $\epsilon>0$, there exists a point $b_{\epsilon} \in B$ such that $\omega_{\lambda}\left(a, b_{\epsilon}\right) \leq \Omega_{\lambda}(A, B)+\epsilon$.

Proof. Let $a \in A, \epsilon, \lambda>0$ be arbitrary. Since $\omega_{\lambda}(a, B)=\inf _{b \in B} \omega_{\lambda}(a, b)$, we claim that $\omega_{\lambda}(a, B)+\epsilon$ is not a lower bound of the set $\left\{\omega_{\lambda}(a, b): b \in B\right\}$. Therefore, there exists $b_{\epsilon} \in B$ for which $\omega_{\lambda}\left(a, b_{\epsilon}\right) \leq \omega_{\lambda}(a, B)+\epsilon$ and hence $\omega_{\lambda}\left(a, b_{\epsilon}\right) \leq \Omega_{\lambda}(A, B)+\epsilon$.

\section{Fixed-Point Theorems for Multivalued Mappings}

In this section, we extend the result by Mongkolkeha et al. [14] under the multivalued setting and hereby obtain some corollaries. Beforehand, we will give the notion of a multivalued $\omega$-contraction in modular metric spaces.

Definition 3.1. Let $X_{\omega}$ be a modular metric space. A multivalued mapping $F: X_{\omega} \rightarrow \mathcal{C B}\left(X_{\omega}\right)$ is said to be a multivalued $\omega$-contraction if there exists $0 \leq k<1$ such that

$$
\Omega_{\lambda}(F x, F y) \leq k \omega_{\lambda}(x, y)
$$

for all $x, y \in X_{\omega}$ and $\lambda>0$. In this case, the least number $k$ which satisfies the inequality (3.1) is said to be the contraction constant.

Remark 3.2. For a sequence $\left\{x_{n}\right\}_{n \in \mathbb{N}}$ in $X_{\omega}$, it is obvious that, if $\lim _{n \rightarrow+\infty} x_{n}=x$ and $F$ is a multivalued $\omega$-contraction on $X_{\omega}$, then $\lim _{n \rightarrow+\infty} F x_{n}=F x$.

Theorem 3.3. Let $X_{\omega}$ be a complete modular metric space and $F$ a multivalued $\omega$-contraction on $X_{\omega}$ with contraction constant $k$. Then, $F$ has a fixed point in $X_{\omega}$.

Proof. Let $x_{0} \in X_{\omega}$ be arbitrary and $x_{1} \in F x_{0}$. By Lemma 2.5, there exists $x_{2} \in F x_{1}$ such that

$$
\omega_{\lambda}\left(x_{1}, x_{2}\right) \leq \Omega_{\curlywedge}\left(F x_{0}, F x_{1}\right)+k .
$$

Similarly, by this procedure, we define a sequence $\left\{x_{n}\right\}_{n \in \mathbb{N}}$ in $X_{\omega}$ such that $x_{n} \in F x_{n-1}$ and

$$
\omega_{\lambda}\left(x_{n}, x_{n+1}\right) \leq \Omega_{\lambda}\left(F x_{n-1}, F x_{n}\right)+k^{n}
$$

for all $n \in \mathbb{N}$. Hence, by the multivalued $\omega$-contractivity, we have

$$
\begin{aligned}
\omega_{\lambda}\left(x_{n}, x_{n+1}\right) & \leq \Omega_{\lambda}\left(F x_{n-1}, F x_{n}\right)+k^{n} \\
& \leq k \omega_{\lambda}\left(x_{n-1}, x_{n}\right)+k^{n} \\
& \leq k\left[k \omega_{\lambda}\left(x_{n-2}, x_{n-1}\right)+k^{n-1}\right]+k^{n} \\
& \leq k^{2} \omega_{\lambda}\left(x_{n-2}, x_{n-1}\right)+2 k^{n} .
\end{aligned}
$$


Thus, by induction, we deduce that

$$
\omega_{\lambda}\left(x_{n}, x_{n+1}\right) \leq k^{n} \omega_{\lambda}\left(x_{0}, x_{1}\right)+n k^{n}
$$

Notice that $\sum_{n \in \mathbb{N}} k^{n}<+\infty$ and $\sum_{n \in \mathbb{N}} n k^{n}<+\infty$. Now, since

$$
\sum_{n \in \mathbb{N}} \omega_{\lambda}\left(x_{n}, x_{n+1}\right) \leq \omega_{\lambda}\left(x_{0}, x_{1}\right) \sum_{n \in \mathbb{N}} k^{n}+\sum_{n \in \mathbb{N}} n k^{n}<+\infty
$$

for all $\lambda>0$. Without loss of generality, suppose $m, n \in \mathbb{N}$ and $m>n$. Observe that, for arbitrary $\epsilon>0$,

$$
\begin{aligned}
\omega_{\lambda}\left(x_{n}, x_{m}\right) & \leq \omega_{\lambda /(m-n)}\left(x_{n}, x_{n+1}\right)+\omega_{\lambda /(m-n)}\left(x_{n+1} n, x_{n+2}\right)+\cdots+\omega_{\lambda /(m-n)}\left(x_{m-1}, x_{m}\right) \\
& \leq \omega_{\lambda / m}\left(x_{n}, x_{n+1}\right)+\omega_{\lambda / m}\left(x_{n+1} n, x_{n+2}\right)+\cdots+\omega_{\lambda / m}\left(x_{m-1}, x_{m}\right) \\
& \leq \sum_{n=n_{*}}^{+\infty} \omega_{\lambda / m}\left(x_{n}, x_{n+1}\right) \\
& <\epsilon
\end{aligned}
$$

for all $m>n \geq n_{*}$ for some $n_{*} \in \mathbb{N}$, and hence $\left\{x_{n}\right\}_{n \in \mathbb{N}}$ is a Cauchy sequence. Then, the completeness of $X_{\omega}$ implies that $\lim _{n \rightarrow+\infty} x_{n}=x$ for some $x \in X_{\omega}$. Consequently, the sequence $\left\{F x_{n}\right\}_{n \in \mathbb{N}}$ converges to $F x$, that is, $\lim _{n \rightarrow+\infty} \Omega_{\lambda}\left(F x_{n}, F x\right)=0$ for all $\lambda>0$. Since $x_{n} \in F x_{n-1}$, we have

$$
0 \leq \omega_{\lambda}\left(x_{n+1}, F x\right) \leq \delta_{\lambda}\left(F x_{n}, F x\right) \leq \Omega_{\curlywedge}\left(F x_{n}, F x\right)
$$

which implies that $\omega_{\lambda}(x, F x)=0$. Since $F x$ is closed, it follows that $x \in F x$.

Example 3.4. Let $X=[0,1], \omega:(0,+\infty) \times X \times X \rightarrow[0,+\infty]$ defined by $\omega_{\lambda}(x, y):=(1 /(1+\lambda)) \mid x-$ $y \mid$. Clearly, $X_{\omega}=[0,1]$ for any generator $x_{\iota} \in X$. Now, we define a multivalued mapping $F: X_{\omega} \rightarrow \mathcal{C B}\left(X_{\omega}\right)$ given by

$$
F x=\left\{0, \frac{x+1}{2}\right\} .
$$

We have $\Omega_{\lambda}(F x, F y)=(1 / 2(1+\lambda))|x-y| \leq(1 / 2) \omega_{\lambda}(x, y)$. Therefore, $F$ is a multivalued $\omega$-contraction with contraction constant $k=1 / 2$, and we have that 0 and 1 are fixed points of F.

Remark 3.5. Note that our result does not assure the uniqueness of a fixed point, as illustrated in the above example.

We next present the local version of Theorem 3.3. 
Theorem 3.6. Let $X_{\omega}$ be a complete modular metric space,

$$
\mathbb{B}_{\omega}\left(x_{0}, \gamma\right):=\left\{x \in X_{\omega}: \omega_{\lambda}\left(x, x_{0}\right) \leq \gamma, \forall \lambda>0\right\},
$$

and $F: \mathbb{B}_{\omega}\left(x_{0}, \gamma\right) \rightarrow \mathcal{C} B\left(X_{\omega}\right)$. Suppose there exists $0 \leq k<1$ for which

$$
\Omega_{\lambda}(F x, F y) \leq k \omega_{\lambda}(x, y)
$$

for all $x, y \in B_{\omega}\left(x_{0}, \gamma\right), \lambda>0$ and

$$
\Omega_{\curlywedge}\left(F x_{0},\left\{x_{0}\right\}\right) \leq(1-k) \gamma
$$

for all $\lambda>0$. Then, $F$ has a fixed point in $\boldsymbol{B}_{\omega}\left(x_{0}, \gamma\right)$.

Proof. To prove this theorem, we only need to show that $B_{\omega}\left(x_{0}, \gamma\right)$ is complete and $F x \subseteq$ $B_{\omega}\left(x_{0}, \gamma\right)$. To show that $B_{\omega}\left(x_{0}, \gamma\right)$ is complete, suppose that $\left\{x_{n}\right\}_{n \in \mathbb{N}}$ is a Cauchy sequence in $\mathbb{B}_{\omega}\left(x_{0}, \gamma\right)$. Since $X_{\omega}$ is complete, $\lim _{n \rightarrow+\infty} \omega_{\lambda}\left(x_{n}, x\right)=0$ for some $x \in X_{\omega}$ for all $\lambda>0$. Since, for each $n \in \mathbb{N}, x_{n} \in \mathbb{B}_{\omega}\left(x_{0}, \gamma\right)$, we get

$$
\begin{aligned}
\omega_{\lambda}\left(x_{0}, x\right) & \leq \omega_{\lambda / 2}\left(x_{0}, x_{n}\right)+\omega_{\lambda / 2}\left(x_{n}, x\right) \\
& \leq \gamma+\omega_{\lambda / 2}\left(x_{n}, x\right) .
\end{aligned}
$$

As $n \rightarrow+\infty$, we have $\omega_{\lambda}\left(x_{0}, x\right) \leq \gamma$. Therefore, $\mathcal{B}_{\omega}\left(x_{0}, \gamma\right)$ is complete.

Now, we prove the latter. For any $x \in B_{\omega}\left(x_{0}, \gamma\right)$, let $y \in F x$. Observe that, for all $\lambda>0$,

$$
\begin{aligned}
\omega_{\lambda}\left(y, x_{0}\right) & =\delta_{\lambda}\left(\{y\},\left\{x_{0}\right\}\right) \\
& \leq \delta_{\lambda / 3}(\{y\}, F x)+\delta_{\lambda / 3}\left(F x, F x_{0}\right)+\delta_{\lambda / 3}\left(F x_{0},\left\{x_{0}\right\}\right) \\
& \leq \Omega_{\lambda / 3}\left(F x, F x_{0}\right)+\Omega_{\lambda / 3}\left(F x_{0},\left\{x_{0}\right\}\right) \\
& \leq k \omega_{\lambda}\left(x, x_{0}\right)+(1-k) \gamma \\
& \leq \gamma .
\end{aligned}
$$

This implies that $F x \subseteq \boldsymbol{B}_{\omega}\left(x_{0}, \gamma\right)$ for all $x \in \mathcal{B}_{\omega}\left(x_{0}, \gamma\right)$. Applying Theorem 3.3 to complete the proof.

In the following theorem, we prove the existence of fixed points for a mapping introduced in 1969 by Kannan [15] in view of multivalued mappings in modular metric spaces.

Theorem 3.7. Let $X_{\omega}$ be a complete modular metric space and $F: X_{\omega} \rightarrow \mathcal{C B}\left(X_{\omega}\right)$ a multivalued mapping such that there exists $0 \leq k<1 / 2$ such that

$$
\Omega_{\lambda}(F x, F y) \leq k\left[\omega_{2 \lambda}(x, F x)+\omega_{2 \lambda}(y, F y)\right]
$$

for all $x, y \in X_{\omega}$ and $\lambda>0$. Then, $F$ has a fixed point in $X_{\omega}$. 
Proof. Let the sequence $\left\{x_{n}\right\}_{n \in \mathbb{N}}$ be constructed as in the proof of Theorem 3.3, so we get, for all $\lambda>0$,

$$
\omega_{\lambda}\left(x_{n}, x_{n+1}\right) \leq \Omega_{\lambda}\left(F x_{n-1}, F x_{n}\right)+k^{n}
$$

for all $n \in \mathbb{N}$. Observe that

$$
\begin{aligned}
\omega_{\lambda}\left(x_{n}, x_{n+1}\right) & \leq \Omega_{\lambda}\left(F x_{n-1}, F x_{n}\right)+k^{n} \\
& \leq k\left[\omega_{2 \lambda}\left(x_{n-1}, F x_{n-1}\right)+\omega_{2 \lambda}\left(x_{n}, F x_{n}\right)\right]+k^{n} \\
& \leq k\left[\omega_{\lambda}\left(x_{n-1}, F x_{n-1}\right)+\omega_{\lambda}\left(x_{n}, F x_{n}\right)\right]+k^{n} \\
& \leq k\left[\omega_{\lambda}\left(x_{n-1}, F x_{n-1}\right)+\omega_{\lambda}\left(x_{n}, x_{n+1}\right)\right]+k^{n} .
\end{aligned}
$$

Further, set $\xi:=k /(1-k)<1$, we obtain

$$
\begin{aligned}
\omega_{\lambda}\left(x_{n}, x_{n+1}\right) \leq & \xi \omega_{\lambda}\left(x_{n-1}, x_{n}\right)+\frac{k^{n}}{1-k} \\
& \leq \xi^{2} \omega_{\lambda}\left(x_{n-2}, x_{n-1}\right)+\frac{k^{n}}{(1-k)^{2}}+\frac{k^{n}}{(1-k)} \\
& \leq \xi^{2} \omega_{\lambda}\left(x_{n-2}, x_{n-1}\right)+2 \frac{k^{n}}{(1-k)^{2}} \\
& \vdots \\
& \leq \xi^{n} \omega_{\lambda}\left(x_{0}, x_{1}\right)+n \xi^{n} .
\end{aligned}
$$

As in the proof of Theorem 3.3, we conclude that $\left\{x_{n}\right\}_{n \in \mathbb{N}}$ is a Cauchy sequence. The completeness of $X_{\omega}$ implies that $\lim _{n \rightarrow+\infty} x_{n}=x$ for some $x \in X_{\omega}$.

Now, we show that $x$ is a fixed point of $F$. Observe that

$$
\begin{aligned}
\omega_{\lambda}(x, F x) & =\delta_{\lambda}(\{x\}, F x) \\
& \leq \delta_{\lambda / 2}\left(\{x\}, F x_{n}\right)+\delta_{\lambda / 2}\left(F x_{n}, F x\right) \\
& =\omega_{\lambda / 2}\left(x, F x_{n}\right)+\delta_{\lambda / 2}\left(F x_{n}, F x\right) \\
& \leq \omega_{\lambda / 2}\left(x, x_{n+1}\right)+\Omega_{\lambda / 2}\left(F x_{n}, F x\right) \\
& \leq \omega_{\lambda / 2}\left(x, x_{n+1}\right)+k\left[\omega_{\lambda}\left(x_{n}, F x_{n}\right)+\omega_{\lambda}(x, F x)\right] .
\end{aligned}
$$

Again, we have that

$$
\omega_{\lambda}(x, F x) \leq \frac{1}{1-k} \omega_{\lambda / 2}\left(x, x_{n+1}\right)+\frac{k}{1-k} \omega_{\lambda}\left(x_{n}, F x_{n}\right)
$$

As $n \rightarrow+\infty$, we have $\omega_{\lambda}(x, F x)=0$. Since $F x$ is closed, we have $x \in F x$. Therefore, $x$ is a fixed point of $F$ in $X_{\omega}$. 


\section{Stability of Fixed Points}

In this section, we discuss some stability of fixed points in Theorems 3.3 and 3.7. In this context, $\operatorname{Fix}(F)$ will denote the set of all fixed points of a self-mapping $F$ on $X_{\omega}$.

Theorem 4.1. Let $X_{\omega}$ be a complete modular metric space, and let $F, G: X_{\omega} \rightarrow \mathcal{C B}\left(X_{\omega}\right)$ be two multivalued $\omega$-contractions having the same contraction constant $k$. If, for any $A, B \in \mathcal{C} B\left(X_{\omega}\right)$, $\lim _{\lambda \downarrow} \Omega_{\lambda}(A, B)=\zeta_{(A, B)}<+\infty$, then $\Omega_{\lambda}(\operatorname{Fix}(F), \operatorname{Fix}(G)) \leq(1-k)^{-1} \sup _{x \in X_{\omega}} \zeta_{(F x, G x)}$.

Proof. Suppose $\lambda>0$, by Theorem 3.3, we can conclude that $\operatorname{Fix}(F) \neq \emptyset \neq \operatorname{Fix}(G)$. Let $\epsilon>0$ be arbitrary, and let $\gamma>0$ be such that $\gamma \sum_{n \in \mathbb{N}} n k^{n}<1$. For $x_{0} \in \operatorname{Fix}(F)$, choose $x_{1} \in G x_{0}$ such that

$$
\omega_{\lambda}\left(x_{0}, x_{1}\right) \leq \Omega_{\lambda}\left(F x_{0}, G x_{0}\right)+\epsilon .
$$

By the multivalued $\omega$-contractivity, it is possible to choose $x_{2} \in G x_{1}$ such that

$$
\omega_{\lambda}\left(x_{1}, x_{2}\right) \leq k \omega_{\lambda}\left(x_{0}, x_{1}\right)+\frac{\gamma \epsilon k}{1-k} .
$$

Now, define a sequence $\left\{x_{n}\right\}_{n \in \mathbb{N}}$ inductively by $x_{n} \in G x_{n-1}$ and

$$
\omega_{\lambda}\left(x_{n}, x_{n+1}\right) \leq k \omega_{\lambda}\left(x_{n-1}, x_{n}\right)+\frac{\gamma \epsilon k^{n}}{1-k} .
$$

Set $\eta:=\gamma \epsilon /(1-k)$, it follows that

$$
\begin{aligned}
\omega_{\lambda}\left(x_{n}, x_{n+1}\right) & \leq k \omega_{\lambda}\left(x_{n-1}, x_{n}\right)+\eta k^{n} \\
& \leq k^{2} \omega_{\lambda}\left(x_{n-1}, x_{n-1}\right)+2 \eta k^{n} .
\end{aligned}
$$

Inductively, we have that

$$
\omega_{\lambda}\left(x_{n}, x_{n+1}\right) \leq k^{n} \omega_{\lambda}\left(x_{0}, x_{1}\right)+n \eta k^{n} .
$$

Notice that $\sum_{n \in \mathbb{N}} k^{n}<+\infty$ and $\sum_{n \in \mathbb{N}} n k^{n}<+\infty$. Now, since

$$
\sum_{n \in \mathbb{N}} \omega_{\lambda}\left(x_{n}, x_{n+1}\right) \leq \omega_{\lambda}\left(x_{0}, x_{1}\right) \sum_{n \in \mathbb{N}} k^{n}+\eta \sum_{n \in \mathbb{N}} n k^{n}<+\infty,
$$

we can say that $\left\{x_{n}\right\}_{n \in \mathbb{N}}$ is a Cauchy sequence. The completeness of $X_{\omega}$ implies that $\lim _{n \rightarrow+\infty} x_{n}=x$ for some $x \in X_{\omega}$. Since $\lim _{n \rightarrow+\infty} \Omega_{\lambda}\left(G x_{n}, G x\right)=0$ and $x_{n} \in G x_{n-1}$, we get $x \in \operatorname{Fix}(G)$. Now, observe that

$$
\begin{aligned}
\omega_{\lambda}\left(x_{0}, x\right) & \leq \omega_{\lambda / 2 n}\left(x_{0}, x_{1}\right)+\omega_{\lambda / 2 n}\left(x_{1}, x_{2}\right)+\cdots+\omega_{\lambda / 2 n}\left(x_{n-1}, x_{n}\right)+\omega_{\lambda / 2}\left(x_{n}, x\right) \\
& \leq \sum_{m \in \mathbb{N}} \omega_{\lambda / 2 n}\left(x_{m-1}, x_{m}\right)+\omega_{\lambda / 2}\left(x_{n}, x\right) \\
& \leq \omega_{\lambda / 2 n}\left(x_{0}, x_{1}\right) \sum_{m \in \mathbb{N}} k^{m-1}+\eta \sum_{m \in \mathbb{N}}(m-1) k^{m-1}+\omega_{\lambda / 2}\left(x_{n}, x\right) \\
& \leq(1-k)^{-1}\left[\omega_{\lambda / 2 n}\left(x_{0}, x_{1}\right)+\epsilon\right]+\omega_{\lambda / 2}\left(x_{n}, x\right) \\
& \leq(1-k)^{-1}\left[\Omega_{\lambda / 2 n}\left(F x_{0}, G x_{0}\right)+2 \epsilon\right]+\omega_{\lambda / 2}\left(x_{n}, x\right) .
\end{aligned}
$$


Since $\omega_{\lambda}\left(x_{0}, x\right) \geq \omega_{\lambda}\left(x_{0}, \operatorname{Fix}(G)\right)$ and together with (4.18), we have, as $n \rightarrow+\infty$, that

$$
\begin{aligned}
\delta_{\lambda}(\operatorname{Fix}(F), \operatorname{Fix}(G)) & \leq(1-k)^{-1}\left[\sup _{x \in \operatorname{Fix}(F)} \zeta_{(F x, G x)}+2 \epsilon\right] \\
& \leq(1-k)^{-1}\left[\sup _{x \in X_{\omega}} \zeta_{(F x, G x)}+2 \epsilon\right] .
\end{aligned}
$$

Similarly, we have

$$
\delta_{\lambda}(\operatorname{Fix}(G), \operatorname{Fix}(F)) \leq(1-k)^{-1}\left[\sup _{x \in X_{\omega}} \zeta_{(F x, G x)}+2 \epsilon\right] .
$$

Since $\epsilon>0$ is arbitrary, this completes the proof.

Corollary 4.2. Let $X_{\omega}$ be a complete modular metric space and $F_{n}: X_{\omega} \rightarrow \mathcal{C B}\left(X_{\omega}\right)$, for $n \in \mathbb{N}$, multivalued $\omega$-contractions having the same contraction constant $k$, and for any $A, B \in \mathcal{C B}\left(X_{\omega}\right)$, $\lim _{\lambda \downarrow 0} \Omega_{\lambda}(A, B)=\zeta_{(A, B)}<+\infty$. If $\lim _{n \rightarrow+\infty} \zeta_{\left(F_{n} x, F x\right)}=0$ uniformly for $x \in X_{\omega}$, then $\lim _{n \rightarrow+\infty} \Omega_{\lambda}\left(\operatorname{Fix}\left(F_{n}\right), \operatorname{Fix}(F)\right)=0$.

Proof. Let $\epsilon>0$ be arbitrary. Since $\lim _{n \rightarrow+\infty} \zeta_{\left(F_{n} x, F x\right)}=0$ uniformly for $x \in X_{\omega}$ and $\lambda>0$, there exists $n_{0} \in \mathbb{N}$ such that

$$
\sup _{x \in X_{\omega}} \zeta_{\left(F_{n} x, F x\right)}<(1-k) \epsilon
$$

for all $n \in \mathbb{N}$ with $n \geq n_{0}$. By Theorem 4.1 , we have

$$
\Omega_{\lambda}\left(\operatorname{Fix}\left(F_{n}\right), \operatorname{Fix}(F)\right)<\epsilon
$$

for all $n \in \mathbb{N}$ with $n \geq n_{0}$ and $\lambda>0$.

Likewise, we can deduce a stability theorem for fixed points in Theorem 3.7.

Theorem 4.3. Let $X_{\omega}$ be a complete modular metric space, and let $F, G: X_{\omega} \rightarrow \mathcal{C B}\left(X_{\omega}\right)$ be two multivalued mappings such that there exists $0 \leq k<1$ such that

$$
\begin{aligned}
& \Omega_{\lambda}(F x, F y) \leq k\left[\omega_{2 \lambda}(x, F x)+\omega_{2 \lambda}(y, F y)\right], \\
& \Omega_{\lambda}(G x, G y) \leq k\left[\omega_{2 \lambda}(x, G x)+\omega_{2 \lambda}(y, G y)\right],
\end{aligned}
$$

for all $x, y \in X_{\omega}$ and $\lambda>0$. If, for any $A, B \in \mathcal{C} B\left(X_{\omega}\right), \lim _{\lambda \downarrow 0} \Omega_{\lambda}(A, B)=\zeta_{(A, B)}<+\infty$, then $\Omega_{\lambda}(\operatorname{Fix}(F), \operatorname{Fix}(G)) \leq(1-k)^{-1} \sup _{x \in X_{\omega}} \zeta_{(F x, G x)}$. 
Proof. Suppose $\lambda>0$, by Theorem 3.7, we can conclude that $\operatorname{Fix}(F) \neq \emptyset \neq \operatorname{Fix}(G)$. Let $\epsilon>0$ be arbitrary, and let $\gamma>0$ be such that $\gamma \sum_{n \in \mathbb{N}} n k^{n}<1$. For $x_{0} \in \operatorname{Fix}(F)$, choose $x_{1} \in G x_{0}$ such that

$$
\omega_{\lambda}\left(x_{0}, x_{1}\right) \leq \Omega_{\lambda}\left(F x_{0}, G x_{0}\right)+\frac{\gamma \epsilon}{1-k} .
$$

It is possible to choose $x_{2} \in G x_{1}$ such that

$$
\omega_{\lambda}\left(x_{1}, x_{2}\right) \leq \Omega_{\curlywedge}\left(G x_{0}, G x_{1}\right)+\frac{\gamma \epsilon}{1-k} k
$$

By induction, we can construct a sequence $\left\{x_{n}\right\}_{n \in \mathbb{N}}$ such that

$$
\omega_{\lambda}\left(x_{n}, x_{n+1}\right) \leq \Omega_{\lambda}\left(G x_{n-1}, G x_{n}\right)+\frac{\gamma \epsilon}{1-k} k^{n} .
$$

Observe that

$$
\begin{aligned}
\omega_{\lambda}\left(x_{n}, x_{n+1}\right) & \leq \Omega_{\lambda}\left(F x_{n-1}, F x_{n}\right)+\frac{\gamma \epsilon}{1-k} k^{n} \\
& \leq k\left[\omega_{2 \lambda}\left(x_{n-1}, F x_{n-1}\right)+\omega_{2 \lambda}\left(x_{n}, F x_{n}\right)\right]+\frac{\gamma \epsilon}{1-k} k^{n} \\
& \leq k\left[\omega_{\lambda}\left(x_{n-1}, F x_{n-1}\right)+\omega_{\lambda}\left(x_{n}, F x_{n}\right)\right]+\frac{\gamma \epsilon}{1-k} k^{n} \\
& \leq k\left[\omega_{\lambda}\left(x_{n-1}, F x_{n-1}\right)+\omega_{\lambda}\left(x_{n}, x_{n+1}\right)\right]+\frac{\gamma \epsilon}{1-k} k^{n} .
\end{aligned}
$$

Further, set $\xi:=k /(1-k)<1$ and $\eta:=\gamma \epsilon /(1-k)$, we obtain

$$
\begin{aligned}
\omega_{\lambda}\left(x_{n}, x_{n+1}\right) \leq & \xi \omega_{\lambda}\left(x_{n-1}, x_{n}\right)+\frac{\gamma \epsilon}{(1-k)^{2}} k^{n} \\
\leq & \xi^{2} \omega_{\lambda}\left(x_{n-2}, x_{n-1}\right)+2 \frac{\gamma \epsilon}{(1-k)^{3}} k^{n} \\
& \vdots \\
\leq & \xi^{n} \omega_{\lambda}\left(x_{0}, x_{1}\right)+n \eta \xi^{n} .
\end{aligned}
$$

Similar to the proof of Theorem 4.1, we conclude that $\left\{x_{n}\right\}_{n \in \mathbb{N}}$ is a Cauchy sequence. The completeness of $X_{\omega}$ implies that $\left\{x_{n}\right\}_{n \in \mathbb{N}}$ converges to some limit $x \in X_{\omega}$. We can further see that $x \in \operatorname{Fix}(G)$. Now, observe that

$$
\begin{aligned}
\omega_{\lambda}\left(x_{0}, x\right) & \leq \omega_{\lambda / 2 n}\left(x_{0}, x_{1}\right)+\omega_{\lambda / 2 n}\left(x_{1}, x_{2}\right)+\cdots+\omega_{\lambda / 2 n}\left(x_{n-1}, x_{n}\right)+\omega_{\lambda / 2}\left(x_{n}, x\right) \\
& \leq \sum_{m \in \mathbb{N}} \omega_{\lambda / 2 n}\left(x_{m-1}, x_{m}\right)+\omega_{\lambda / 2}\left(x_{n}, x\right) \\
& \leq \omega_{\lambda / 2 n}\left(x_{0}, x_{1}\right) \sum_{m \in \mathbb{N}} k^{m-1}+\eta \sum_{m \in \mathbb{N}}(m-1) k^{m-1}+\omega_{\lambda / 2}\left(x_{n}, x\right) \\
& \leq(1-k)^{-1}\left[\omega_{\lambda / 2 n}\left(\mathrm{x}_{0}, x_{1}\right)+\epsilon\right]+\omega_{\lambda / 2}\left(x_{n}, x\right) \\
& \leq(1-k)^{-1}\left[\Omega_{\lambda / 2 n}\left(F x_{0}, G x_{0}\right)+2 \epsilon\right]+\omega_{\lambda / 2}\left(x_{n}, x\right) .
\end{aligned}
$$


Since $\omega_{\lambda}\left(x_{0}, x\right) \geq \omega_{\lambda}\left(x_{0}, \operatorname{Fix}(G)\right)$ and together with (4.18), we have, as $n \rightarrow+\infty$, that

$$
\begin{aligned}
\delta_{\lambda}(\operatorname{Fix}(F), \operatorname{Fix}(G)) & \leq(1-k)^{-1}\left[\sup _{x \in \operatorname{Fix}(F)} \zeta_{(F x, G x)}+2 \epsilon\right] \\
& \leq(1-k)^{-1}\left[\sup _{x \in X_{\omega}} \zeta_{(F x, G x)}+2 \epsilon\right] .
\end{aligned}
$$

Similarly, we have

$$
\delta_{\lambda}(\operatorname{Fix}(G), \operatorname{Fix}(F)) \leq(1-k)^{-1}\left[\sup _{x \in X_{\omega}} \zeta_{(F x, G x)}+2 \epsilon\right] .
$$

Since $\epsilon>0$ is arbitrary, this completes the proof.

Corollary 4.4. Let $X_{\omega}$ be a complete modular metric space, and let $F_{n}: X_{\omega} \rightarrow \mathcal{C B}\left(X_{\omega}\right)$, for $n \in \mathbb{N}$, be multivalued mappings such that there exists $0 \leq k<1$ such that

$$
\Omega_{\lambda}\left(F_{n} x, F_{n} y\right) \leq k\left[\omega_{2 \lambda}\left(x, F_{n} x\right)+\omega_{2 \lambda}\left(y, F_{n} y\right)\right]
$$

for all $x, y \in X_{\omega}$ and $\lambda>0$. Suppose for any $A, B \in \mathcal{C B}\left(X_{\omega}\right), \lim _{\downarrow_{\downarrow} 0} \Omega_{\lambda}(A, B)=\zeta_{(A, B)}<+\infty$. If $\lim _{n \rightarrow+\infty} \zeta_{\left(F_{n} x, F x\right)}=0$ uniformly for $x \in X_{\omega}$, then $\lim _{n \rightarrow+\infty} \Omega_{\lambda}\left(\operatorname{Fix}\left(F_{n}\right), \operatorname{Fix}(F)\right)=0$.

Proof. Let $\epsilon>0$ be arbitrary. Since $\lim _{n \rightarrow+\infty} \zeta_{\left(F_{n} x, F x\right)}=0$ uniformly for $x \in X_{\omega}$ and $\lambda>0$, there exists $n_{0} \in \mathbb{N}$ such that

$$
\sup _{x \in X_{\omega}} \zeta_{\left(F_{n} x, F x\right)}<(1-k) \epsilon
$$

for all $n \in \mathbb{N}$ with $n \geq n_{0}$. By Theorem 4.3 , we have

$$
\Omega_{\lambda}\left(\operatorname{Fix}\left(F_{n}\right), \operatorname{Fix}(F)\right)<\epsilon
$$

for all $n \in \mathbb{N}$ with $n \geq n_{0}$ and $\lambda>0$.

\section{Acknowledgments}

The authors were supported by the Higher Education Research Promotion and National Research University Project of Thailand, Office of the Higher Education Commission (NRUCSEC no. 54000267). The second author was supported by the Thailand Research Fund through the Royal Golden Jubilee Ph.D. program (Grant no. PHD/0029/2553). The third author would like to thank the Research Professional Development Project Under the Science Achievement Scholarship of Thailand (SAST). The authors are very grateful to the referees for the valuable suggestions and comments. This work is dedicated to Professor Sompong Dhompongsa with admiration and respect. 


\section{References}

[1] S. Banach, "Sur les opérations dans les ensembles abstraits et leur application aux équations intégrales," Fundamenta Mathematicae, vol. 3, p. 160, 1922.

[2] S. B. Nadler, Jr., "Multi-valued contraction mappings," Pacific Journal of Mathematics, vol. 30, pp. 475$488,1969$.

[3] Z. Liu, Y. Xu, and Y. J. Cho, "Common fixed point theorems for single-valued and multi-valued mappings," Publicationes Mathematicae Debrecen, vol. 61, no. 3-4, pp. 439-453, 2002.

[4] R. P. Agarwal, Y. J. Cho, and D. O’Regan, "Fixed point and homotopy invariant results for multivalued maps on complete guage spaces," Bulletin-Australian Mathematical Society, vol. 67, pp. 241248, 2003.

[5] Y. Song and Y. J. Cho, "Iterative approximations for multivalued nonexpansive mappings in reflexive Banach spaces," Mathematical Inequalities E Applications, vol. 12, no. 3, pp. 611-624, 2009.

[6] N. Hussain, A. Amini-Harandi, and Y. J. Cho, "Approximate endpoints for set-valued contractions in metric spaces," Fixed Point Theory and Applications, vol. 2010, Article ID 614867, 13 pages, 2010.

[7] M. Abbas and Y. J. Cho, "Fixed point results for multi-valued non-expansive mappings on an unbounded set," Analele Stiintifice ale Universitatii Ovidius Constanta, vol. 18, no. 2, pp. 5-14, 2010.

[8] Y. J. Cho, S. Hirunworakit, and N. Petrot, "Set-valued fixed-point theorems for generalized contractive mappings without the Hausdorff metric," Applied Mathematics Letters, vol. 24, no. 11, pp. 1959-1967, 2011.

[9] W. Sintunavarat and P. Kumam, "Common fixed point theorem for hybrid generalized multi-valued contraction mappings," Applied Mathematics Letters, vol. 25, no. 1, pp. 52-57, 2012.

[10] K. Kuaket and P. Kumam, "Fixed points of asymptotic pointwise contractions in modular spaces," Applied Mathematics Letters, vol. 24, no. 11, pp. 1795-1798, 2011.

[11] C. Mongkolkeha and P. Kumam, "Fixed point and common fixed point theorems for generalized weak contraction mappings of integral type in modular spaces," International Journal of Mathematics and Mathematical Sciences, vol. 2011, Article ID 705943, 12 pages, 2011.

[12] C. Mongkolkeha and P. Kumam, "Fixed point theorems for generalized asymptoticpointwise $\rho$-contraction mappings involving orbits in modular function spaces," Applied Mathematics Letters. In press.

[13] V. V. Chistyakov, "Modular metric spaces, I: basic concepts," Nonlinear Analysis: Theory, Methods and Applications, vol. 72, no. 1, pp. 1-14, 2010.

[14] C. Mongkolkeha, W. Sintunavarat, and P. Kumam, "Fixed point theorems for contraction mappings in modular metric spaces," Fixed Point Theory and Applications, vol. 2011, article 93, 2011.

[15] R. Kannan, "Some results on fixed points. II," The American Mathematical Monthly, vol. 76, pp. 405-408, 1969. 


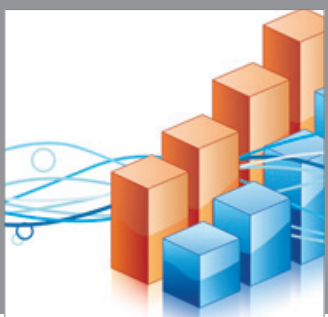

Advances in

Operations Research

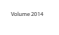

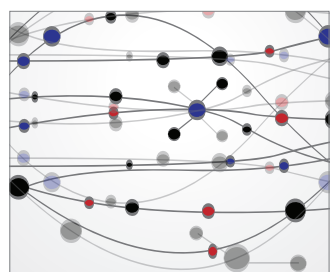

\section{The Scientific} World Journal
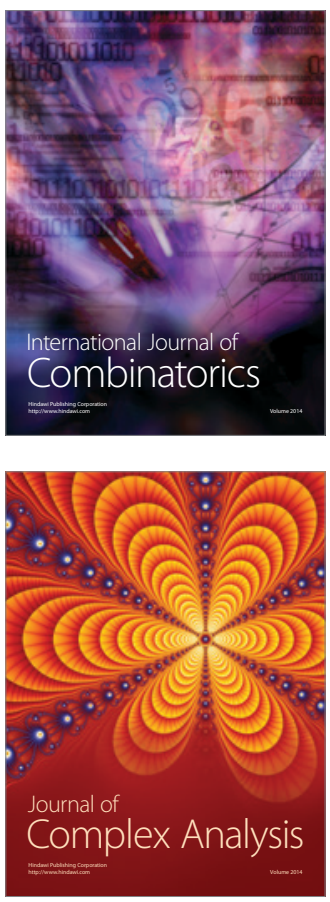

International Journal of

Mathematics and

Mathematical

Sciences
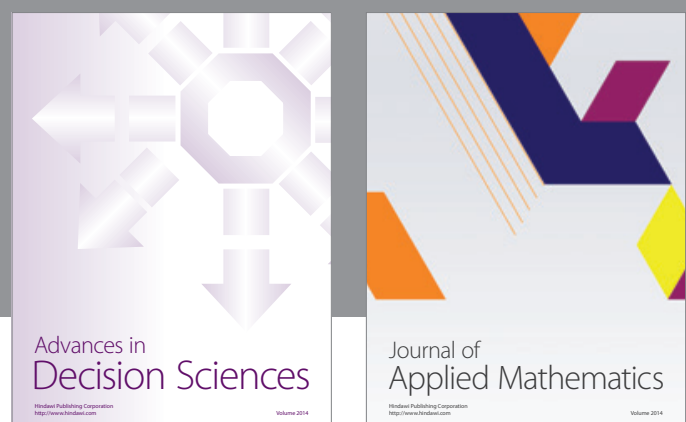

Journal of

Applied Mathematics
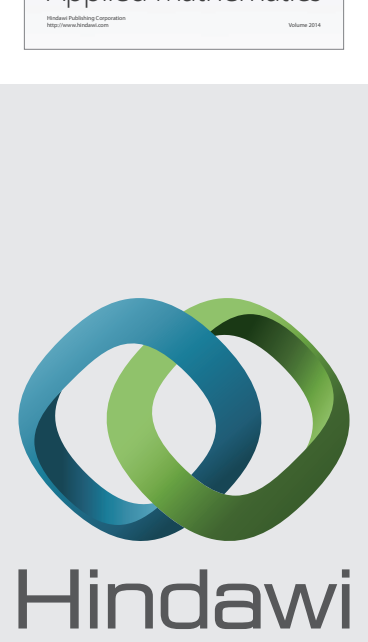

Submit your manuscripts at http://www.hindawi.com
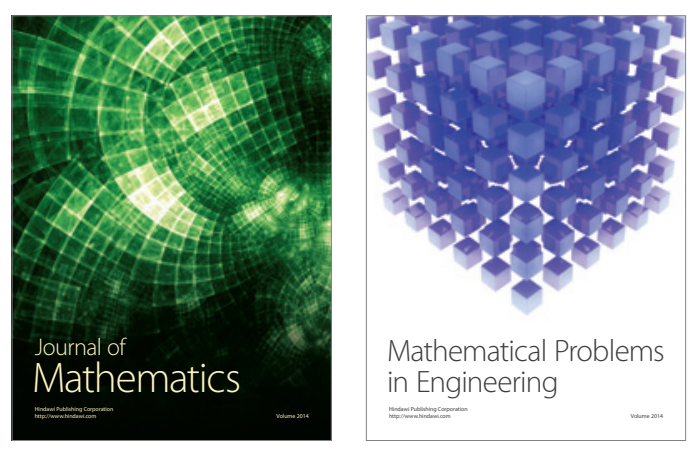

Mathematical Problems in Engineering
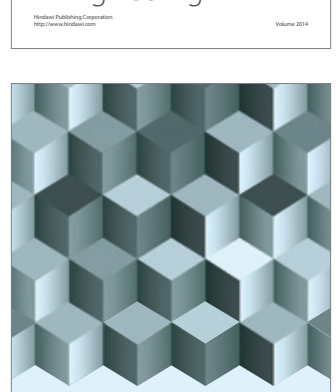

Journal of

Function Spaces
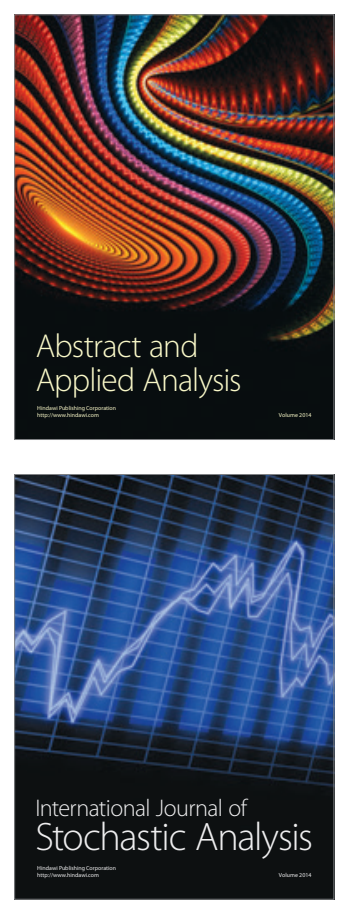

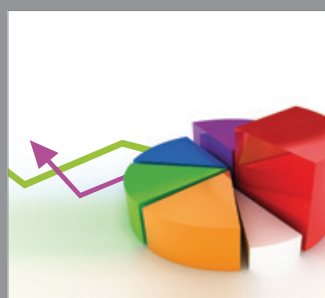

ournal of

Probability and Statistics

Promensencen
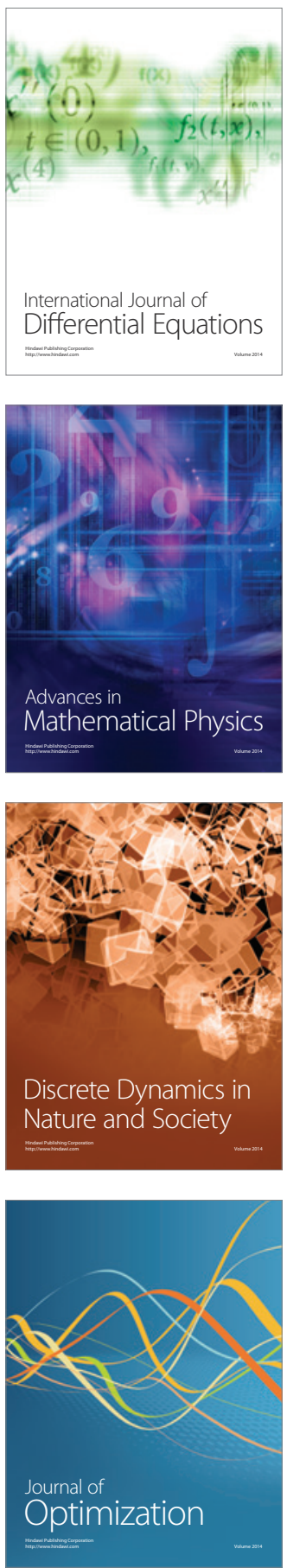